\title{
When Dogs Shrink the Typical Lengthening Effect Caused by Negative Emotions
}

\author{
Quentin Hallez®e, Nicolas Baltenneck, and Anna-Rita Galiano
}

Department of Psychology, Unité de recherche DIPHE (Développement Individu Processus Handicap Education), University Lumiere Lyon 2, Bron, France

\begin{abstract}
This paper examines how dogs can modulate the effects of emotion on time perception. To this end, participants performed a temporal bisection task with stimulus durations presented in the form of neutral or emotional facial expressions (angry, sad, and happy faces). In the first experiment, dog owners were compared with nondog owners, while in the second experiment, students were randomly assigned to one of the three waiting groups (waiting alone, with another person, or with a dog) before being confronted with the temporal bisection task. The results showed that dogs allowed the participants to regulate the intensity of negative emotional effects, while no statistical differences emerged for the happy facial expressions. In certain circumstances, dogs could even lead the subjects to generate underestimation of time when faced with negative facial expressions.
\end{abstract}

Keywords: time, emotion, animal mediation, regulation, timing

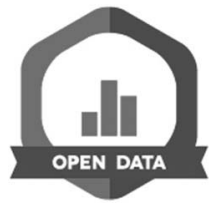

The last decade has seen an explosion in research into the effect of emotion on the perception of time (for a recent review, see Droit-Volet et al., 2013). Most of these studies have used facial expressions as emotional stimuli along with a temporal bisection task (Bar-Haim et al., 2010; Doi \& Shinohara, 2009; Droit-Volet et al., 2004; Droit-Volet \& Meck, 2007; Gil \& Droit-Volet, 2011a, 2011b; Tipples, 2008, 2011). In this famous task, participants first have to learn to discriminate a short $(S)$ from a long $(L)$ standard duration based on the presentation of a pink oval shape. Then, in the testing phase, three to five intermediate durations are integrated along with the two previous standard durations. Participants are not aware of this change. They must continue to say "short" or "long" if they think they are faced with the short or long standard duration. In these different studies, negative, high-arousal stimuli, namely angry or fearful faces, are systematically judged to last longer than neutral stimuli. Positive facial expressions (e.g., happy faces) or negative but lowarousing facial expressions (e.g., sad faces) were also proved to last longer compared to neutral faces (DroitVolet et al., 2004; Droit-Volet \& Meck, 2007; Gil \& DroitVolet, 2011a, 2011b). Nonetheless, the lengthening effect matched with these emotions is often deemed to be less important. In summary, the perception of emotional stimuli, specifically the negative high arousal stimuli, typically produces distortions in time judgment consistent with a "lengthening effect."

This phenomenon has been explained by researchers in light of the most popular model of time perception - the pacemaker-accumulator-like model (Gibbon et al., 1984; Treisman, 1963). According to the pacemaker-accumulator model, each individual is intrinsically equipped with an "internal clock" which itself comprises a pacemaker, an attentional switch, and an accumulator. The pacemaker emits pulses to an accumulator via an attentional switch that closes at the beginning and opens at the end of the stimulus to be timed. Our perception of time would therefore rely on the time units incremented in the accumulator. The more pulses accumulated, the longer the subjective time. Thereby, based on this model, the lengthening effect would be the result of the perception of negative high-arousal emotional stimuli increasing the level of activation of the central nervous system and thus accelerating the internal clock system underlying the representation of time (Gil \& Droit-Volet, 2012; Treisman, 1963, 2013). This assumption has been confirmed with pharmacological studies in which participants were administered dopamine-releasing substances such as cocaine or methamphetamine that modulate arousal by altering the effective levels of dopamine in the brain (Cheng et al., 2007; Matell et al., 2006 for a 
review of the effects of dopamine on time perception, also see Marinho et al., , 2018). Just as a clock speeds up, arousal would increase the ticking rate of our internal clock. The main function of this would be to enable the body to adapt efficiently to forthcoming events (DroitVolet \& Meck, 2007). For example, fear and anger are extremely arousing as they activate behavior fundamental to our survival (e.g., fight or flee). Sadness or happiness, however, needs social responses that are less linked to bodily survival.

There is some evidence that emotional distortions in duration perception can be modified. For instance, the temporal bias caused by emotion can be modulated according to the chosen external emotional stimuli. Indeed, the lengthening effect witnessed with an emotional stimulus as simple as a movie (Droit-Volet et al., 2011) becomes more marked with stronger emotional stimuli such as electric shocks or aversive sounds for instance, since these last stimuli are more arousing (Droit-Volet et al., 2010; Fayolle et al., 2015, also see Gil \& DroitVolet, 2012). Yet, the effect can also be modified by changing the subject's receptivity to these distractors. In a recent study, researchers demonstrated that a cognitive re-evaluation (e.g., reappraisal) and an inhibition of our own emotion expression (e.g., suppression) were emotional regulation strategies that drastically reduced (or even canceled) the influence of emotional facial expressions on time perception (Tian et al., 2018). It is to highlight that reappraisal and suppression have been shown to decrease emotional arousal and even change brain activity related to emotional processing (Cai et al., 2016; Driscoll et al., 2009; Eippert et al., 2007; Flynn et al., 2010; Goldin et al., 2008), therefore explaining the aforementioned effects.

To our knowledge, no studies have attempted to analyze the influence of an animal on the temporal bias related to emotions. Yet, among the vast array of physiological and psychological benefits achieved with dogs (e.g., reduced blood pressure, reduced heart rate, diminished sense of loneliness, and reduced pain threshold: Anderson et al., 1992; Polheber \& Matchock, 2014; Zasloff \& Kidd, 1994; Petersson et al., 1996), one such benefit is linked with arousal. Indeed, it has been shown that the presence of or prior interaction with a dog decreases autonomic arousal (Jennings, 1997). For instance, Allen et al. analyzed autonomic responses in women while performing a backward subtraction task. The women could be alone, with a female friend, or with a pet dog. Participants in the latter scenario presented lower heart rate, skin conductance, and blood pressure compared to the other two groups (Allen et al., 1991). Regardless of the situations and individuals tested (i.e., gender and age), participants systematically report feeling less stressed in canine company compared to other individuals (Hansen et al., 1999; Siegel, 1990). The factor behind these lower arousal rates is thought to be related to a decrease in cortisol production. Cortisol is directly related to stress since this hormone is actively produced during stressful situations (for a review, see Burke et al., 2005). In a stressful situation, cortisol transforms fat into sugar so that the body has the necessary energy to react to the danger (De Feo et al., 1989). Many studies have thus shown that a 10-min interaction with a dog significantly reduces cortisol levels in individuals (Handlin et al., 2011; Pendry \& Vandagriff, 2019; Viau et al., 2010). The same effects were observed in dog owners (Allen et al., 2002), where the cortisol levels of these subjects are associated with the way the owners interact with their dogs and also with behaviors caused by the interaction (Petersson et al., 2017).

The aim of our study was therefore to systematically analyze the possible modulation that dogs could generate on emotion-related time distortion. In the first study of this paper, we directly compared dog owners to individuals without animals. Our hypotheses were that (1) dog owners should display fewer time distortions associated with emotional events, (2) especially for high-arousing facial expressions, regardless of their positive or negative valence. It was also assumed that (3) these effects would be linked to the amount of time dog owners spent with their dogs.

\section{Experiment 1}

\section{Method}

\section{Participants}

A total of 130 subjects took part in the experiment. Among them, 103 were dog owners (93 females and 10 males, $M_{\text {age }}=35.99$ years, $S D=12.19$ ) and 27 were not ( 20 females and seven males, $M_{\text {age }}=33.5, S D=13.87$ ). The participants were enrolled in the study via an advertisement posted publicly on Facebook as well as in private groups of dog enthusiasts. Since these private groups are breed-specific, we targeted the owners of medium and large dogs. Informed written consent was obtained from all participants prior to participation.

\section{Material}

The survey was conducted using Qualtrics ${ }^{\circledast}$ electronic survey software, which delivered and encoded experimental events. The temporal stimuli were a pink oval image $(12 \times 16 \mathrm{~cm})$ and photos of three different female faces (Droit-Volet et al., 2004), retrieved from the Radboud Faces Database (Langner et al., 2010). Temporal stimuli were 
always displayed in the center of the screen against a black background. Four different facial expressions were paired with each of the women (i.e., Neutral, Sadness, Happiness, and Anger; see an example in Figure 1).

\section{Procedure}

Before starting the task, participants were invited to answer demographic questions about gender and age and to confirm whether they were dog owners. If they owned a $\mathrm{dog}$, they were asked how much interaction time they had with their dog during the day and how much interaction time they had with their dog on average. Interaction was defined as reciprocal reactions and moments of exchange.

Participants were then invited to perform a temporal bisection task composed of three successive phases: pretraining, training, and testing. In the pretraining phase, participants were submitted to "short" and "long" standard duration twice, represented by the pink oval stimulus. The short standard stimulus duration was $1,000 \mathrm{~ms}$, and the long standard stimulus was 4,000 ms. Instructions were also given before the presentation, specifying the duration to be presented (i.e., "you will now see the short duration") and after the presentation, recalling the temporal stimulus they were just shown (i.e., "this was the short duration"). Participants were also asked not to count as this is an effective method used to prevent counting (Rattat \& Droit-Volet, 2012).

In the training phase, participants were exposed to a block of eight trials, with standard durations each

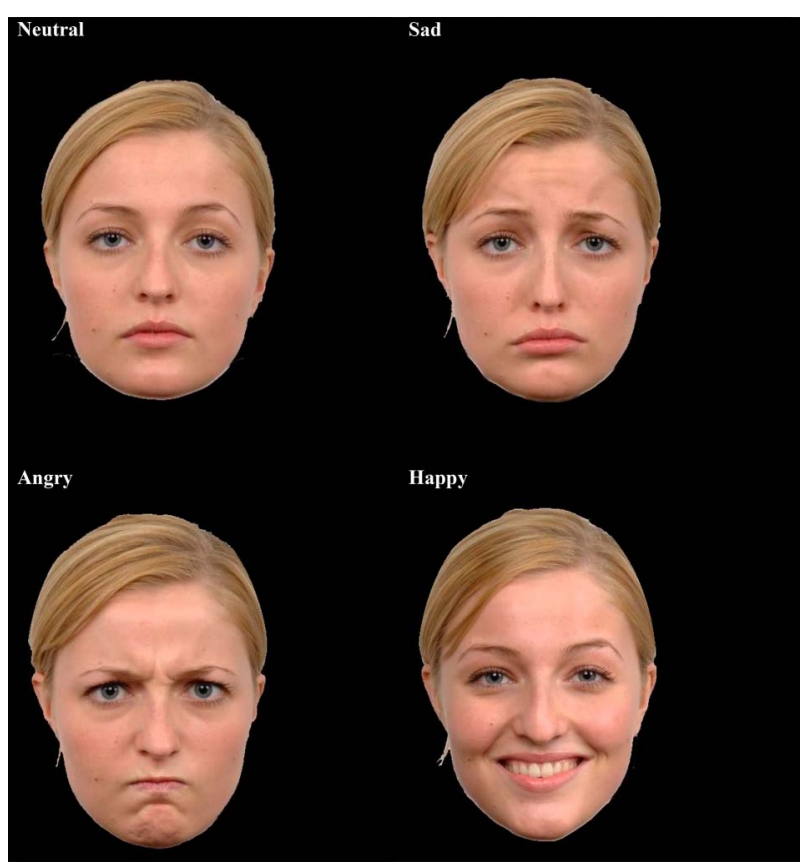

Figure 1. Example of faces displaying neutral, sad, angry and happy expressions. randomly presented four times. Following each standard duration, participants had to choose responses labeled "short" or "long" displayed on the survey screen. They therefore had to discriminate between durations by ticking "short" after the short standard duration, or "long," after the long standard duration. Participants could not proceed to the next presentation without responding. Accuracy feedback was then presented in the center of the screen for $2 \mathrm{~s}$, with "yes" or "no" for correct and incorrect answers, respectively.

During the testing phase, the temporal stimuli took the form of an emotional facial expression, thus replacing the pink oval image. No further accuracy feedbacks were delivered. Subjects were informed of these changes. However, they were not warned that three intermediate durations had been inserted (e.g., 1,750, 2,500, and $3,250 \mathrm{~ms}$ ) along with the two standard durations. Their objective was unchanged; they had to tick "short" or "long" when they thought they had been exposed to the short or long duration. Subjects thus underwent a total of 180 trials (four expressions $\times$ three female faces $\times$ three trials $\times$ five durations).

\section{Results}

\section{Data Analysis}

Figure 2 shows the proportion of "long" responses ( $p$ (long)) plotted against the comparison durations for each emotional condition for both dog owners and nondog owners (Figure 2). Also, Table 1 provides the bisection point (BP) and Weber ratio (WR) for each experimental condition for both Experiments 1 and 2. Graphically, it appears that the psychophysical function of the nondog owners was shifted toward the left, consistent with a lengthening effect, for the angry and sad faces compared to the neutral faces. Nonetheless, the psychophysical functions of dog owners seem to remain consistent throughout. This was confirmed by the statistical analysis run on $p$ (long). Notwithstanding the significant results, we will not report them here as they generated similar results to those obtained for the BP. The BP is the "psychological mid-point" of the duration range between $S$ and $L$ (Wearden, 2016, p. 72). In other words, it is the duration giving rise to $50 \%$ of "short" responses and $50 \%$ of "long" responses. This temporal parameter was derived from the significant fit of the individual data obtained with the pseudologistic model (PLM; Killeen et al., 1997), which provides good fits for the bisection data under the different conditions (mean $\mathrm{R}^{2}=.91, S D=0.04$ ). In addition, Wearden and Ferrara (1995) demonstrated that the results were quite similar, irrespective of the method used. Another parameter, the WR, was calculated. The WR is an index of time sensitivity. It is the difference limen $(p$ (long $)=.75-p($ long $)=.25)$ 
A

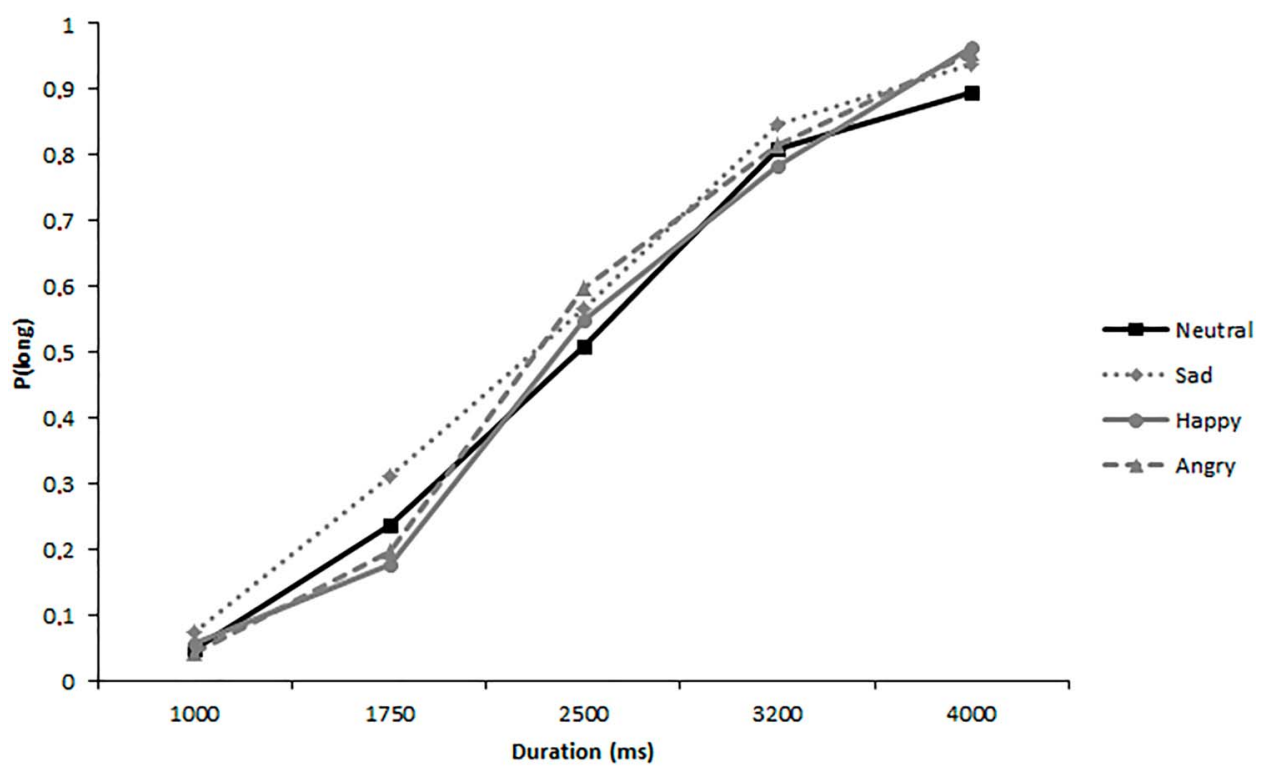

Figure 2. Proportion of long responses plotted against probe durations for the neutral, sad, happy, and angry facial expressions for both (panel A) Dog Owners and (panel B) Nondog owners.

B

\section{No Dog Owners}

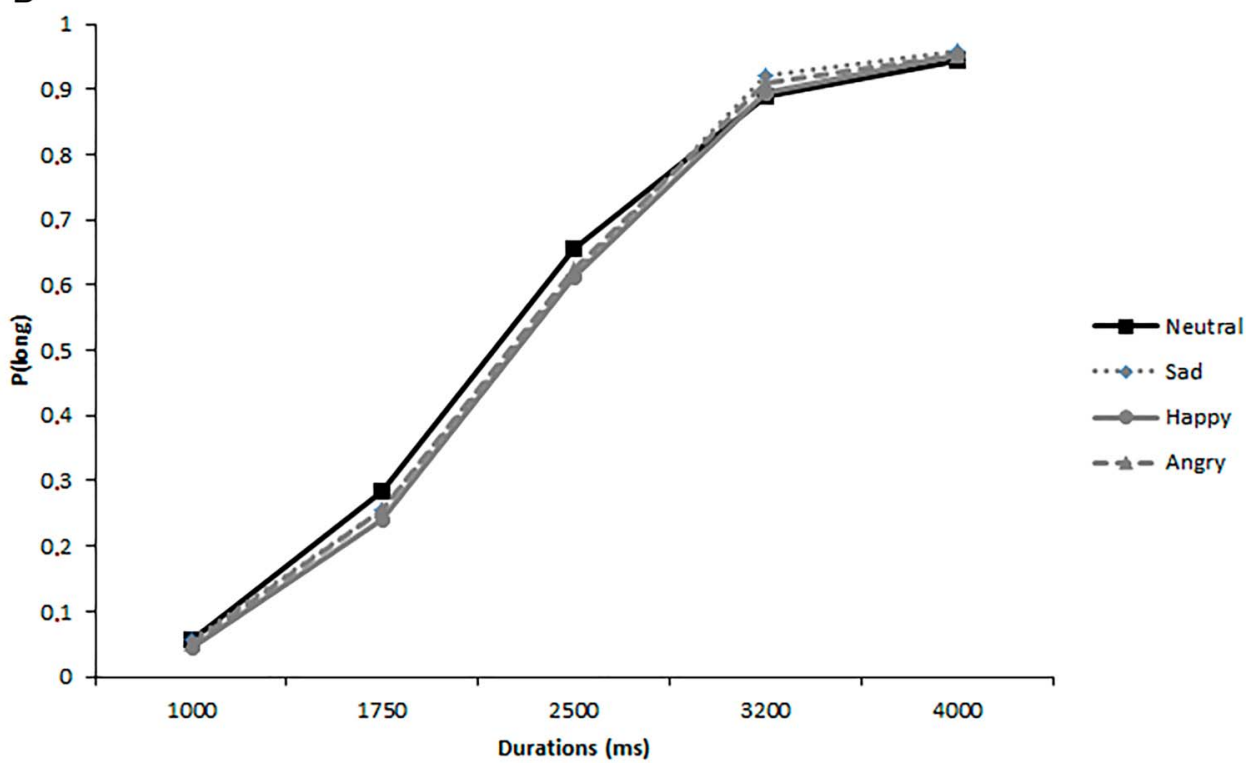

divided by the BP. A high WR indicates low time sensitivity, and a low WR is indicative of high time sensitivity. The following link gives access to raw data for Studies 1 and 2: https://osf.io/nehs4/?view_only=0ce9a765c1c442 faa $863 \mathrm{~b} 290 \mathrm{f} 943 \mathrm{fc} 28$.

\section{Weber Ratio and Bisection Point Analyses}

The statistical analyses launched on the WR did not show any significant results (all $p>.05$ ). This therefore suggests that time sensitivity did not change according to the type of facial emotional expression and dog ownership group. This result corroborates previous studies on emotions (see Droit-Volet, 2013, for reviews). Nonetheless, the BP ANOVA with expressions as withinsubject factors (neutral, sad, happy, and angry) and group of dog owners as a between-subject factor (dog owners or nondog owners) revealed a significant emotion $\times$ group interaction, $F(4,126)=3.39, p=.02, \eta^{2} p$ $=.07$. Yet, the ANOVA did not yield any main effect in terms of emotion, $F(4,126)=0.89, p=.45$, or dog ownership, $F(1,129)=3.37, p>.05$. Taken together, these results suggest that the shift of the $\mathrm{BP}$ with faces is 
Table 1. Summary table of BPs and WR for each of the emotional conditions (anger, sadness, happiness, and neutral) for Experiments 1 and 2

\begin{tabular}{|c|c|c|c|c|c|c|c|c|}
\hline & \multicolumn{2}{|c|}{ Anger } & \multicolumn{2}{|c|}{ Sadness } & \multicolumn{2}{|c|}{ Happiness } & \multicolumn{2}{|c|}{ Neutral } \\
\hline & $\mathrm{BP}$ & WR & $\mathrm{BP}$ & WR & $\mathrm{BP}$ & WR & $\mathrm{BP}$ & WR \\
\hline \multicolumn{9}{|l|}{ Experiment 1} \\
\hline No dog owners & 2.37 & 0.42 & 2.30 & 0.46 & 2.45 & 0.44 & 2.53 & 0.50 \\
\hline Dog owners & 2.25 & 0.39 & 2.32 & 0.42 & 2.28 & 0.40 & 2.23 & 0.45 \\
\hline \multicolumn{9}{|l|}{ Experiment 2} \\
\hline Waiting alone & 2.28 & 0.43 & 2.30 & 0.46 & 2.22 & 0.46 & 2.43 & 0.44 \\
\hline Waiting with someone & 2.44 & 0.38 & 2.42 & 0.47 & 2.47 & 0.53 & 2.63 & 0.45 \\
\hline Waiting with a dog & 2.46 & 0.39 & 2.46 & 0.42 & 2.24 & 0.48 & 2.29 & 0.51 \\
\hline
\end{tabular}

the only group function in our study. To ensure effective evaluation of our theoretical predictions, we launched mixed ANOVAs comparing each emotional condition separately with the neutral faces.

\section{Analyses of the Temporal Bias for the Emotion of Anger}

First, we launched the mixed ANOVA on angry faces with one within-factor for emotion (angry and neutral) and one between-subject factor for dog ownership (dog owners and nondog owners). This model did not highlight any main effect on emotion, $F(1,129)=2.19, p=.14$, suggesting that there was no significant distortion in time perception from neutral to angry faces. Nonetheless, the emotion $\times$ group ownership just reached the level of significance, $F(1$, $129)=3.92, p=.05, \eta_{\mathrm{p}}^{2}=.03$. As suggested by Figure 3 , which illustrates the difference in BP from neutral to emotional faces, participants without dogs are more likely to generate a typical lengthening effect caused by angry faces than dog owners. Additional paired $t$-tests were run for each dog ownership group to compare these distortions with zero. While participants without a dog significantly generate the typical lengthening effect $(t(27)=2.0, p=.05)$, distortions among dog owners do not differ statistically from zero $(t(103)=-0.55, p>.05)$. Finally, the significant main effect of the dog ownership yielded by the ANOVA, $F(1,129)=5.57, p=.02, \eta^{2} p=.04$, means that subjects without dogs have a BP shifted further to the right ( $M=2.45 \mathrm{~s})$ compared to dog owners $(M=2.24 \mathrm{~s})$. Thereby, dog owners would underestimate time on the temporal bisection when faced with neutral and angry emotional faces compared to other subjects without dogs.

\section{Analyses of the Temporal Bias for the Emotion of Sadness}

The same ANOVA was run on sad faces. Once again, the ANOVA revealed no main effect of emotion, $F(1$, $129)=1.87, p=.17$, but a significant emotion $\times$ group ownership interaction, $F(1,129)=10.30, p=.002, \eta_{p}^{2}=.07$. When comparing the BP differences from neutral to sad

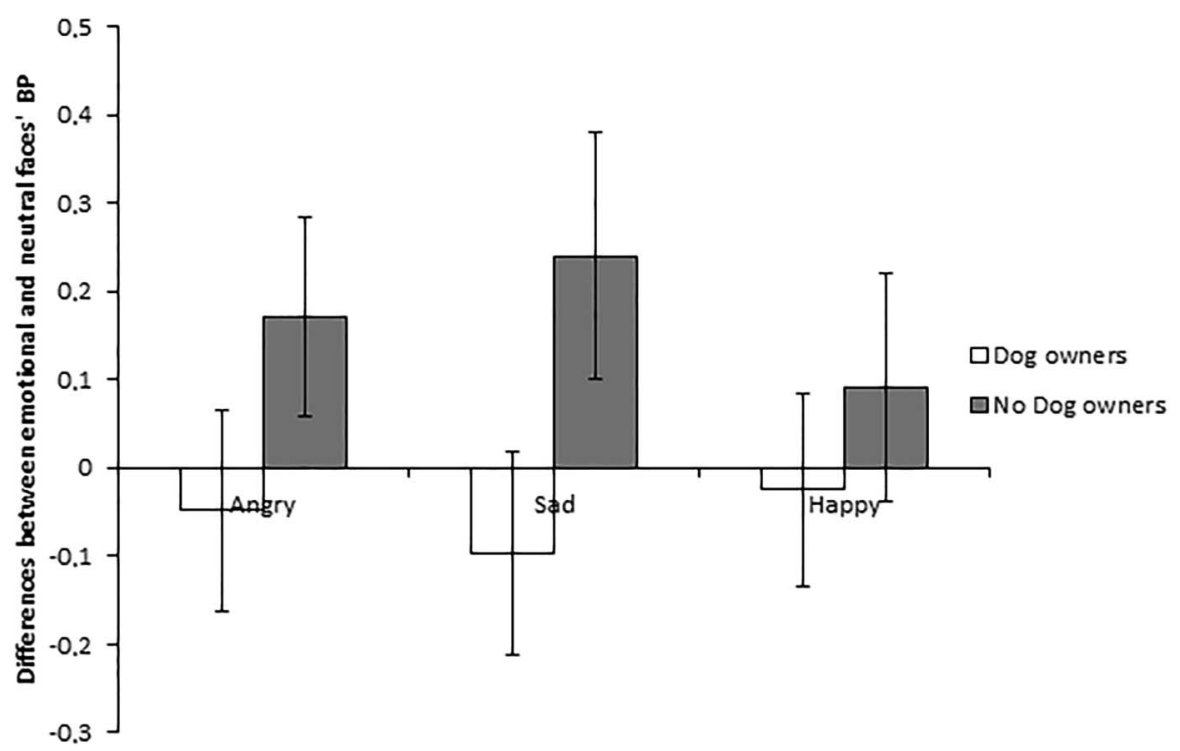

Figure 3. Illustration of the time distortion between neutral and emotional faces for each of our experimental conditions (angry, sad, and happy faces). The error bars show estimation variances divided by two. $\mathrm{BP}=$ bisection point 
faces with zero, it appears that subjects without a dog reproduce the typical lengthening effect caused by emotion $(t(27)=2.22, p=.037)$, while dog owners are significantly generating an underestimation of time $t(103)=-2.11, p=.035)$. The ANOVA yielded no main group effect in this model, $F(1,129)=2.97, p>.05$.

\section{Analyses of the Temporal Bias for the Emotion of Happiness}

Finally, the ANOVA on happy faces only discloses the main effect of group owners, $F(1,129)=7.79, p=.006, \eta^{2} p=.06$, with a lower BP paired with dog owners $\left(M_{\text {dog owners }}=2.25\right.$ s; $M_{\text {nondog owners }}=2.49 \mathrm{~s}$ ). The effects of emotion and emotion $\times$ group both failed to reach the level of significance $(F(1,129)=0.19, p=.67 ; F(1,129)=2.02, p=.16$, respectively).

\section{Regression Analyses of the Interaction Time With Canines Reported by Dog Owners on the Emotional Temporal Estimation Bias}

Finally, to answer our last hypothesis, linear regressions were initiated to establish whether the time spent with their dog can predict the reduced difference in BP from neutral to emotional faces that we observed in dog owners. We used both the average estimated interaction time generally spent by owners with their dogs and the interaction time spent with their dogs the day before taking part in the study as independent variables. The first measure showed no significant regression equation (angry distortions: $F(1,94)=3.72, p=.057$; sadness distortions: $F(1$, $94)=2.60, p=.11$; happiness distortions: $F(1,94)=0.002$, $p=.96)$. However, the regression equation regarding sad distortion proved to be significant with the interaction time subjects spent with their dogs the day before taking part, $F(1,106)=3.94, p=.05$, with an $\eta_{p}^{2}$ of .04 . The participants' distortion of time is equal to $-.252+0.001$ when time interaction is reported in minutes. Participant distortion caused by sad faces thus decreases by $0.001 \mathrm{~s}$ for each minute of interaction with their dog. Yet, equations failed to reach the level of significance for the happy, $F(1$, 106) $=0.05, p=.82$, and angry distortions, $F(1,106)=0.28$, $p=.60$.

\section{Discussion}

In summary, the results of Experiment 1 suggest that the effect of emotional faces is a function of the dog ownership of participants. However, our first hypothesis that dog owners should generate a less marked lengthening effect for high-arousing expressions has only been partially validated. While a greater time-lengthening effect was observed in individuals without a dog compared to individuals with a dog, regarding the angry faces, this was not the case with happy faces. Against our expectations, this reduced lengthening effect of dog owners was even observed in the sad face context. This therefore raises concerns regarding our theoretical hypothesis that the influence of dogs on time perception would be based on reduced arousal. Furthermore, it is worth noting that the underestimation of time observed in dog owners with regard to the sad faces was not related to the average amount of time subjects spend with their dog each day but was a function of the amount of time spent on the day of the experiment. This could therefore suggest that the effect of dogs on temporal distortions is of brief duration.

At this point, skeptics might claim that we do not know if it is the dog that generates these reductions in temporal distortion or whether people least affected by these distortions are more likely to own a dog. Furthermore, having a breed dog may reflect different demographic variables when compared with subjects without dog, such as economic conditions, as a breed dog has a cost to purchase and maintain or even physical condition as dog owners may walk more than those people who have no dogs. In addition, the fact of having carried out the study online can also have consequences in relation to the equipment used by users (e.g., screen size). Given the lack of control over these variables, we initiated a second experiment in which students, irrespective of dog ownership, were assigned to one of the three waiting groups: waiting alone, with another person, or with a dog. The aim of Experiment 2 was thus to replicate the results of Experiment 1 based on a more controlling, systematic approach. Thus, our hypothesis was that subjects who waited with a dog would display the least lengthening effect in response to sad and angry faces compared to the two control groups, namely subjects who waited alone or with another person.

\section{Experiment 2}

\section{Method}

\section{Participants}

Seventy-one subjects took part in this experiment (61 females and 10 males, $M_{\text {age }}=20.33$ years, $\left.S D=3.05\right)$. The participants were recruited via an announcement posted publicly by the university. A dislike of dogs was an exclusion criterion.

\section{Material}

The dog used was a 2.5-year-old Samoyed female. This breed was selected for its sympathetic, comforting 
characteristics. The temporal stimuli used for the bisection task were similar to those of Experiment 1.

\section{Procedure}

Participants were seated in a waiting room and were assigned to one of three waiting groups. They could wait alone, with another unknown person, or with a dog. In the second and third instances, the investigator said that they were free to interact while waiting. The interaction time was exactly $15 \mathrm{~min}$ as several recent studies have reported physiological effects (i.e., subject's salivary cortisol levels) following just $10 \mathrm{~min}$ of interaction with a dog (Handlin et al., 2011; Pendry \& Vandagriff, 2019). The subjects were then invited to enter another room, separately, to complete the temporal bisection task which was identical to that of Experiment 1.

\section{Results}

\section{Data Analysis}

Figure 4 shows the proportion of "long" responses ( $p$ (long)) plotted against the comparison durations for each emotional condition for subjects who waited alone, with another person, or with a dog. As in Experiment 1, the WR analyses did not show any significant effect (all $p>.05)$, suggesting that sensitivity to time remained constant under the different experimental conditions. The ANOVAs on $p$ (long) are not shown because the results were similar to those found for the BP.

Similar to Experiment 1, the BPs were calculated using the PLM (Killeen et al., 1997) and provided good fits for the bisection data under the different conditions (mean $\left.R^{2}=.89, S D=0.08\right)$. A mixed ANOVA was launched on the $\mathrm{BP}$ with emotion as a within-subjects factor (neutral, angry, sad, and happy) and the waiting condition as a between-subject factor (waiting alone, waiting with another person, and waiting with a dog). Once again, the main effect of emotions just failed to reach significance, $F(3,68)=2.15, p=.10$. Interestingly, the effect of emotion was function of the waiting condition, as suggested by the significant emotion $\times$ waiting condition interaction, $F(6$, $68)=2.57, p=.02, \eta_{p}^{2}=.07$. The waiting condition was devoid of main effect, $F(2,68)=1.40, p=.25$. As in Experiment 1 , we then launched a mixed ANOVA for each of the three faces. Note that no main effect of waiting groups was documented in any of these ANOVA (all $p s>.05$ ).

\section{Analyses of the Temporal Bias for the Emotion of Anger}

The ANOVA relating to angry faces, that is, with the within-subjects factor of emotion (neutral BP and angry $\mathrm{BP}$ ) and the waiting condition (waiting alone, waiting with another person, and waiting with a dog), showed no main effect of emotion, $F(1,68)=1.70, p=.20$. As suggested by Figure 5 , which highlights the difference between the neutral and emotional faces, the faces $\times$ waiting condition interaction proved to be significant, $F(2,68)=6.55$, $p=.003, \eta_{p}^{2}=.16$. An additional $t$-test revealed that there were no significant differences between the solo and dual waiting conditions, $t(53)=-0.474, p=.64$, but that subjects who waited with a dog systematically showed reduced time lengthening from neutral to angry faces $(M=-0.16 \mathrm{~s})$ than subjects waiting alone $(M=0.14 \mathrm{~s})$ $t(45)=2.81, p=.008$ or with another person $(M=0.18 \mathrm{~s})$ $t(53)=3.27, p=.003$. In summary, the prior 15-min interaction with a dog significantly reduced the time distortion caused by angry faces when compared to individuals who waited alone or with another person. A paired $t$-test comparing these distortions with zero confirmed the lengthening effect for both waiting control conditions $(t(27)=2.19, p=.038 ; t(28)=3.07, p=.005$, respectively). Of even greater interest, participants who interacted with a dog did not showed lengthening effect and even showed underestimation effect, $t(18)=-3.07$, $p=.005$.

\section{Analyses of the Temporal Bias for the Emotion of Sadness}

Similar mixed ANOVA was launched on sad faces and reports similar results. Indeed, the ANOVA did not yield a main effect of emotion, $F(1,68)=1.12, p=.29$, but a significant emotion $\times$ waiting condition interaction, $F(2$, $68)=4.81, p=.011, \eta^{2} p=.12$. Once again, only subjects who waited with a dog differed from the other groups in terms of a significantly reduced lengthening effect $(M=-0.17 \mathrm{~s})$ compared to subjects who waited alone $(M=0.20 \mathrm{~s})$ $t(44)=3.07, p=.004$, or with another person $(M=0.12 \mathrm{~s})$ $t(44)=2.41, p=.021$. No differences emerged between the two control groups $(t(53)=-1.15, p=.26)$. When comparing the difference on BP from neutral to sad emotional faces with zero, only the participants who waited with another person reported a significant overestimation effect, $t(28)=2.59, p=.02$. The underestimation of time from neutral to sad faces observed with dog owners in Experiment 1 just failed to reach the level of significance in this instance for participants who waited with a dog $(t(18)=-1.84, p=.08)$.

\section{Analyses of the Temporal Bias for the Emotion of Happiness}

The ANOVA with happy facial expression differs from other emotions analyzed so far as the model revealed a main effect of emotion, $F(1,68)=6.62, p=.01$, with a bisection shift toward the left for happy faces compared to neutral faces, thereby indicative of a lengthening effect. It 

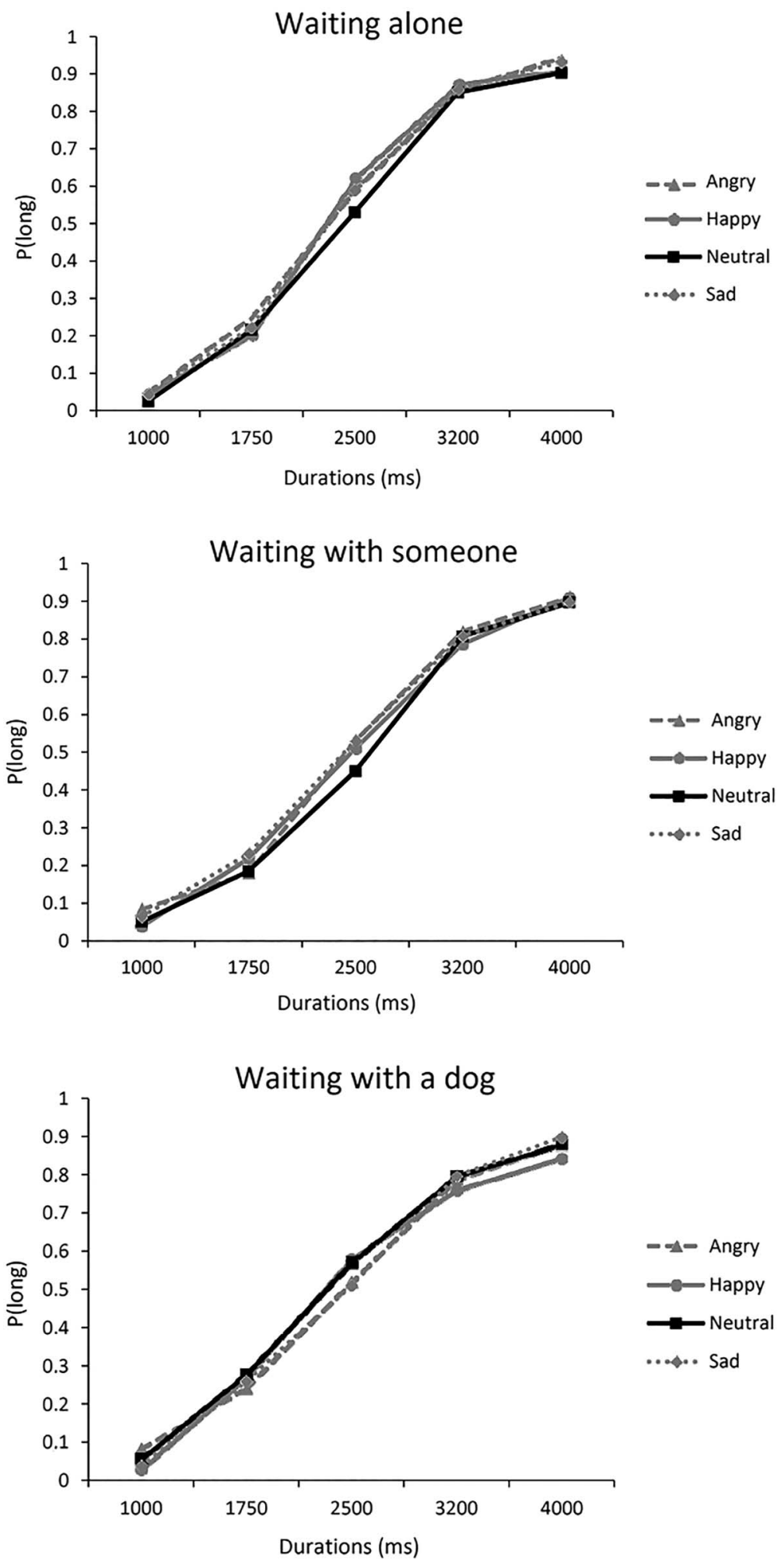

Figure 4. Proportion of long responses plotted against probe durations for the neutral, sad, happy, and angry facial expressions when waiting alone, with another person, and with a dog. 


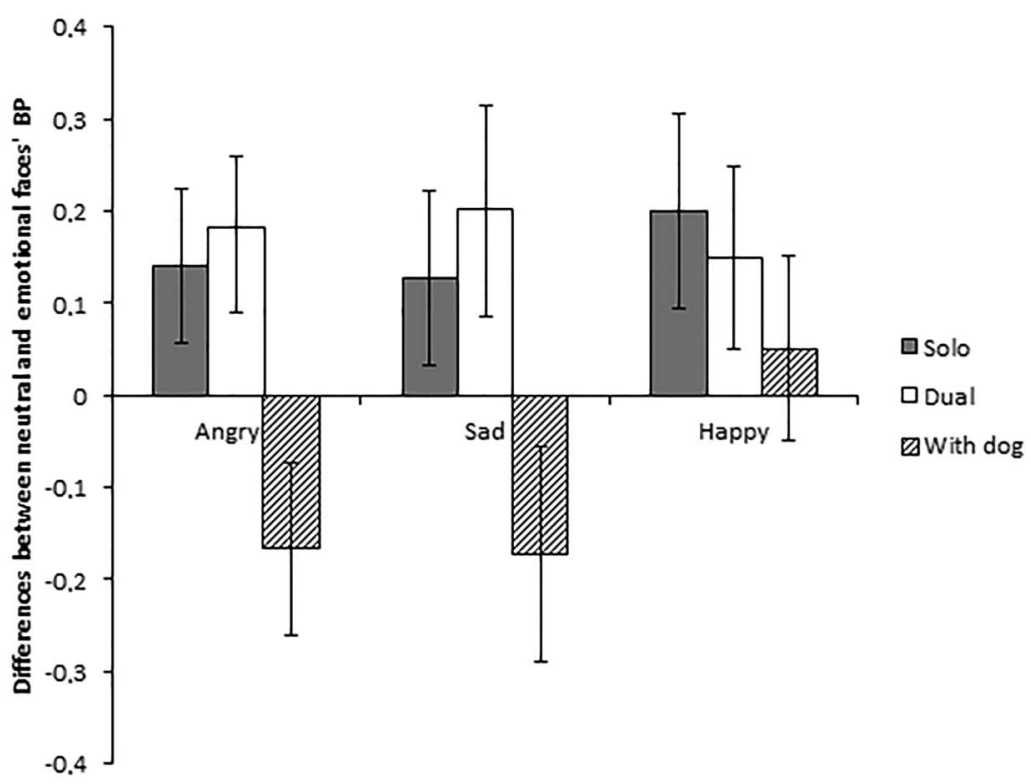

Figure 5. Illustration of the time distortion between neutral and emotional faces for each of our experimental conditions (angry, sad, and happy faces) and waiting condition groups. The error bars show estimation variances divided by two. $\mathrm{BP}=$ bisection point. lengthening effect on exposure to negative facial expressions, namely sad and angry faces. The effect of a dog was sufficiently powerful to eradicate main emotional effect under these conditions. Nonetheless, a lengthening effect was observed in all groups for happy facial expressions, as suggested by the main effect of emotion.

\section{General Discussion}

The aim of our study was to analyze the effect of dogs on typical human time distortion caused by emotional stimuli (e.g., facial expressions) in a temporal bisection task. In our two studies, the results found in our control conditions replicated those reported in numerous studies showing a lengthening effect of perceived durations in response to emotions. Indeed, the psychophysical function obtained in bisection for these subjects was shifted toward the left on exposure to emotional expressions. Both our studies showed that the BP for these subjects was indeed significantly smaller for negative emotional facial expressions (e.g., sad and angry faces) compared to neutral expressions. As the lengthening effect on happy expressions was significant only in Experiment 2, our study also corroborates previous statements announcing that happiness is an emotion which generates fewer temporal distortions (DroitVolet et al., 2004; Droit-Volet \& Meck, 2007; Gil \& DroitVolet, 2011a, 2011b). Furthermore, WRs were devoid of any significant effect in our study, similar to published time and emotion data (see Droit-Volet, 2013). This therefore suggests that time sensitivity does not change according to facial emotional expression and the various groups. This paper thus corroborates recent findings showing no influence of cortisol concentration on empathy and emotion recognition (Duesenberg et al., 2016).

It is particularly interesting to note that our results highlight the important role of dogs in modulating time distortion caused by emotion. Both studies involving dog owners in Experiment 1 and subjects who waited with a dog in Experiment 2 significantly showed a reduced lengthening effect on exposure to both angry and sad faces compared to neutral faces. To our knowledge, this is the first time that such an effect has been reported. Furthermore, Experiment 2 replicates the effects of Experiment 1 with more control over variables, allowing us to conclude with supporting evidence on the existence of this effect. The question that remains to be clarified is that of the mechanism behind the reduction of this "lengthening effect." We first postulated that, because dogs decrease autonomic arousal (Jennings, 1997), a reduced lengthening effect should be observed in relation to high-arousal faces, regardless of their valence. Indeed, according to the pacemaker-accumulator-like model (Gibbon et al., 1984), this overestimation in perceived time results from acceleration of the internal clock rate, associated with an increase in arousal level (Droit-Volet \& Meck, 2007; Gil \& Droit-Volet, 2011a, 2011b). Based on the results associated with the emotional condition of anger, it seems that we can logically assume that dogs decrease autonomic arousal, which consequently prevents or at least reduces modulations in the rhythm of our internal clock.

Nonetheless, if arousal was the only explanation of the influence of dogs on reducing the typical lengthening effect caused by emotion, one could wonder why such an 
effect was not constantly found on happy faces, while it was significant through our two studies upon the emotion of sadness. Yet, it has been demonstrated that happy faces are more arousing than sad faces (Balconi \& Pozzoli, 2009; Balconi \& Lucchiari, 2008). There are two possible explanations for this result. The first explanation could be related with studies having linked the regulation of salivary cortisol with the presence of animals (Handlin et al., 2011; Pendry \& Vandagriff, 2019). These studies showed that, after interacting with a dog for just $10 \mathrm{~min}$, individuals present significantly lower levels of saline cortisol than those having waited alone. As cortisol is the biological response to stress (Mason, 1975; Selye, 1956), the authors have concluded that dogs effectively relieve stress. Thus, the presence of a dog would not decrease the general arousal of the subject but would modulate participants' sensitivity to stress generating stimuli. This is particularly interesting when linked to studies showing connections between stress, anger, and sadness (Martin \& Dahlen, 2005). The fact that the reduction of the temporal bias was linked with the time spent with their dog the day they completed the study is in line with the recent findings of Petersson et al. who demonstrated that cortisol levels of dog owners are directly associated with the way the owners interact with their dogs (Petersson et al., 2017). This hypothesis of a greater emotional regulation associated with negative emotions allowed by the reduction in salivary cortisol could also be in adequacy with the absence of significant differences between our groups regarding the temporal estimations associated with the neutral faces.

The second explanation that could be put forward to explain the lack of the significant influence of dogs on the reduction of the lengthening effect caused by faces of happiness can be related with studies on mood. It is now well documented that a person's mood biases the processing of emotional events, resulting in a processing benefit for mood congruent emotional stimuli. In other words, people who feel and experience an emotion are more likely to be responsive to this specific emotion, which, in turn, can amplify their time distortion in the presence of these specific stimuli. This effect is more commonly known as the mood congruency hypothesis or mood-facilitation hypothesis (for a review, see Droit-Volet et al., 2013). A hypothesis that must therefore be taken into consideration since previous research demonstrated that pet-dog interaction increases positive feelings (Holen, 2012; Le Roux \& Kemp, 2009). Thus, we would not obtain a reduction in the temporal bias caused by the facial expression of happiness following an interaction with a dog because this interaction creates both an increase in the feeling of happiness and a decrease in arousal. In other words, if the more positive mood following the interaction with a dog increases the time lengthening, the effect should be altered, diminished as the reduction in arousal following the interaction with a dog decreases the temporal bias and interacts with the mood effect.

Yet, in a recent study in which fear was induced in participants by film excerpts, the typical temporal overestimation of fearful faces did not occur (Eberhardt et al., 2016). Authors concluded on the importance of dissociating stimulus, state, and trait emotionality to gain a clear understanding of the interplay between emotions and time. Theories of emotion like the state-trait distinction proposed by Cattell and Scheier (1961) differentiate between a "state" from "trait" category as the first reflects a person's current emotions while the second refers to stable characteristics of emotional processing. The moodfacilitation hypothesis was derived from data in which traits between participants were varied, rather than shorter lasting emotional states, i.e., mood. The fact that an underestimation effect was recorded in Experiment 1 for sad expressions while we obtained an underestimation of time for angry faces in Experiment 2 could reflect the distinction made in the literature between state category (dog owners) and trait category (interaction with a dog). That is, the variable influence of dogs in the short (interaction with a dog) or medium/long term (dog owners). Here, a comparison can be made with meditation studies between novices (state) and meditators (traits) showing differences in brain activations, especially in the anterior cingulate cortex and dorsolateral prefrontal areas (Cahn \& Polich, 2006) as well as differences in time perceptions (DroitVolet et al., 2018). The difference could also lay on the nature of the interaction with the dog, which could have been different with an unfamiliar compared with a familiar dog. Since we have not gathered information about the interaction with dogs in both Experiments 1 and 2, we cannot analyze the difference of the interaction subjects may have had with the dog, and we cannot rule on this point.

At this point, it is important to highlight that the original contribution of our study is to reveal the important role that dogs have on our time perception. Yet, as previous studies showed both increases in positive feelings (Holen, 2012; Le Roux \& Kemp, 2009) and decreases of arousal (Jennings, 1997) following the interaction with a pet-dog, it seems necessary in the future to control for these variables to be able to rule on these effects, but also to be able to analyze the interaction between these variables. If future results demonstrate that these effects are caused by the subject's mood, then someone should develop the moodfacilitation hypothesis, distinguishing shorter and lasting emotional states. Irrespective of the causative mechanism, the results of our studies suggest that the effect is shortlasting. Indeed in our first study, the underestimation of time by dog owners exposed to sad facial expressions was 
not linked to the average amount of time spent with dogs in general, but with the length of time spent with their dog on the day of the experiment.

In summary, this paper raises many theoretical questions to be answered in the future by the research community. While the conclusion can be made that dog interaction modulates participants' sensitivity to arousing stimuli, it remains to elude the influence of mood and its possible interactions with arousal, as well as the duration of these effects. This could easily be answered by running specific experimental protocols. Yet and for the first time, our paper reports a reduced lengthening effect in pet owners as well as in subjects who had interacted for just 15 min with an unknown dog, on exposure to negative facial expressions (anger and sadness). This opens up a wide avenue for research. Animal-assisted therapy has become a hotly debated topic in psychology in the past decade because of its broad implications in daily life. It is easily accessible for many populations and is one of the few therapeutic interventions that can continue at home. Finding out more about human-dog interactions and its temporal dynamics could prove to be crucial, especially in a therapeutic context.

\section{References}

Allen, K. M., Blascovich, J., \& Mendes, W. B. (2002). Cardiovascular reactivity and the presence of pets, friends, and spouses: The truth about cats and dogs. Psychosomatic Medicine, 64(5), 727-739. https://doi.org/10.1097/01.psy. 0000024236.11538 .41

Allen, K. M., Blascovich, J., Tomaka, J., \& Kelsey, R. M. (1991). Presence of human friends and pet dogs as moderators of autonomic responses to stress in women. Journal of Personality and Social Psychology, 61(4), 582-589. https://doi.org/10.1037// 0022-3514.61.4.582

Anderson, W. P., Reid, C. M., \& Jennings, G. L. (1992). Pet ownership and risk factors for cardiovascular disease. Medical Journal of Australia, 157(5), 298-301. https://doi.org/10.5694/j.1326-5377. 1992.tb137178.x

Balconi, M., \& Lucchiari, C. (2008). Consciousness and arousal effects on emotional face processing as revealed by brain oscillations: A gamma band analysis. International Journal of Psychophysiology, 67(1), 41-46. https://doi.org/10.1016/j. ijpsycho.2007.10.002

Balconi, M., \& Pozzoli, U. (2009). Arousal effect on emotional face comprehension: Frequency band changes in different time intervals. Physiology \& Behavior, 97(3-4), 455-462. https://doi.org/ 10.1016/j.physbeh.2009.03.023

Bar-Haim, Y., Kerem, A., Lamy, D., \& Zakay, D. (2010). When time slows down: The influence of threat on time perception in anxiety. Cognition and Emotion, 24(2), 255-263. https://doi.org/ 10.1080/02699930903387603

Burke, H. M., Davis, M. C., Otte, C., \& Mohr, D. C. (2005). Depression and cortisol responses to psychological stress: A meta-analysis. Psychoneuroendocrinology, 30(9), 846-856. https://doi.org/10. 1016/j.psyneuen.2005.02.010
Cahn, B. R., \& Polich, J. (2006). Meditation states and traits: EEG ERP, and neuroimaging studies. Psychological Bulletin, 132(2), 180. https://doi.org/10.1037/0033-2909.132.2.180

Cai, A., Yang, J., Xu, S., \& Yuan, J. (2016). The male advantage in regulating negative emotion by expressive suppression: An event-related potential study. Acta Psychologica Sinica, 48(5), 482-494. https://doi.org/J.1041.2016.00482

Cattell, R. B., \& Scheier, I. H. (1961). The meaning and measurement of neuroticism and anxiety. Ronald.

Cheng, R. K., Ali, Y. M., \& Meck, W. H. (2007). Ketamine "unlocks" the reduced clock-speed effects of cocaine following extended training: Evidence for dopamine-glutamate interactions in timing and time perception. Neurobiology of Learning and Memory, 88(2), 149-159. https://doi.org/10.1016/j.nlm.2007.04.005

De Feo, P., Perriello, G., Torlone, E., Ventura, M. M., Fanelli, C., Santeusanio, F., Brunneto, P., Gerich, J. E., \& Bolli, G. B. (1989). Contribution of cortisol to glucose counterregulation in humans. American Journal of Physiology-Endocrinology and Metabolism, 257(1), E35-E42. https://doi.org/10.1152/ajpendo.1989.257.1.E35

Doi, H., \& Shinohara, K. (2009). The perceived duration of emotional face is influenced by the gaze direction. Neuroscience Letters, 457(2), 97-100. https://doi.org/10.1016/j.neulet.2009.04.004

Driscoll, D., Tranel, D., \& Anderson, S. W. (2009). The effects of voluntary regulation of positive and negative emotion on psychophysiological responsiveness. International Journal of Psychophysiology, 72(1), 61-66. https://doi.org/10.1016/j.ijpsycho.2008.03.012

Droit-Volet, S. (2013). Time perception, emotions and mood disorders. Journal of Physiology-Paris, 107(4), 255-264. https://doi. org/10.1016/j.jphysparis.2013.03.005

Droit-Volet, S., Brunot, S., \& Niedenthal, P. (2004). BRIEF REPORT perception of the duration of emotional events. Cognition and Emotion, 18(6), 849-858. https://doi.org/10.1080/02699930341000194

Droit-Volet, S., Chaulet, M., \& Dambrun, M. (2018). Time and meditation: When does the perception of time change with mindfulness exercise? Mindfulness, 9(5), 1557-1570. https://doi. org/10.1007/s12671-018-0903-6

Droit-Volet, S., Fayolle, S. L., \& Gil, S. (2011). Emotion and time perception: Effects of film-induced mood. Frontiers in Integrative Neuroscience, 5, 33. https://doi.org/10.1016/j.sbspro. 2014.02.399

Droit-Volet, S., Fayolle, S., Lamotte, M., \& Gil, S. (2013). Time, emotion and the embodiment of timing. Timing \& Time Perception, 1(1) 99-126. https://doi.org/10.1163/22134468-00002004

Droit-Volet, S., \& Meck, W. H. (2007). How emotions colour our perception of time. Trends in Cognitive Sciences, 11(12), 504-513. https://doi.org/10.1016/j.tics.2007.09.008

Droit-Volet, S., Mermillod, M., Cocenas-Silva, R., \& Gil, S. (2010). The effect of expectancy of a threatening event on time perception in human adults. Emotion, 10(6), 908-914. https://doi.org/10. 1037/a0020258

Duesenberg, M., Weber, J., Schulze, L., Schaeuffele, C., Roepke, S., Hellmann-Regen, J., Otte, C., \& Wingenfeld, K. (2016). Does cortisol modulate emotion recognition and empathy? Psychoneuroendocrinology, 66, 221-227. https://doi.org/10.1016/j. psyneuen.2016.01.011

Eberhardt, L. V., Huckauf, A., \& Kliegl, K. M. (2016). Effects of neutral and fearful mood on duration estimation of neutral and fearful face stimuli. Timing \& Time Perception, 4(1), 30-47. https://doi. org/10.1163/22134468-00002060

Eippert, F., Veit, R., Weiskopf, N., Erb, M., Birbaumer, N., \& Anders, S. (2007). Regulation of emotional responses elicited by threatrelated stimuli. Human Brain Mapping, 28(5), 409-423. https:// doi.org/10.1002/hbm.20291

Fayolle, S., Gil, S., \& Droit-Volet, S. (2015). Fear and time: Fear speeds up the internal clock. Behavioural Processes, 120, 135-140. https://doi.org/10.1016/j.beproc.2015.09.014 
Flynn, J. J., Hollenstein, T., \& Mackey, A. (2010). The effect of suppressing and not accepting emotions on depressive symptoms: Is suppression different for men and women? Personality and Individual Differences, 49(6), 582-586. https://doi. org/10.1016/j.paid.2010.05.022

Gibbon, J., Church, R. M., \& Meck, W. H. (1984). Scalar timing in memory. Annals of the New York Academy of Sciences, 423(1), 52-77. https://doi.org/10.1111/j.1749-6632.1984.tb23417.x

Gil, S., \& Droit-Volet, S. (2011a). How do emotional facial expressions influence our perception of time? In S. Masmoudi, D. Y. Dai, \& A. Naceur (Eds.), Attention, representation, and human performance: integration of cognition, emotion and motivation (pp. 61-74). Psychology Press.

Gil, S., \& Droit-Volet, S. (2011b). "Time flies in the presence of angry faces" depending on the temporal task used!. Acta Psychologica, 136(3), 354-362. https://doi.org/10.1016/j.actpsy.2010.12.010

Gil, S., \& Droit-Volet, S. (2012). Emotional time distortions: The fundamental role of arousal. Cognition and Emotion, 26(5), 847-862. https://doi.org/10.1080/02699931.2011.625401

Goldin, P. R., McRae, K., Ramel, W., \& Gross, J. J. (2008). The neural bases of emotion regulation: Reappraisal and suppression of negative emotion. Biological Psychiatry, 63(6), 577-586. https:// doi.org/10.1016/j.biopsych.2007.05.031

Handlin, L., Hydbring-Sandberg, E., Nilsson, A., Ejdebäck, M., Jansson, A., \& Uvnäs-Moberg, K. (2011). Short-term interaction between dogs and their owners: Effects on oxytocin, cortisol, insulin and heart rate-An exploratory study. Anthrozoös, 24(3), 301-315. https://doi.org/10.2752/175303711X13045914865385

Hansen, K. M., Messinger, C. J., Baun, M. M., \& Megel, M. (1999). Companion animals alleviating distress in children. Anthrozoös, 12(3), 142-148. https://doi.org/10.2752/089279399787000264

Holen, R. (2012). The effects of animal interaction on happiness. Sentience, The University of Minnesota Undergraduate Journa of Psychology, 7, 5-8. https://2538b660-a-551982af-s-sites. googlegroups.com/a/umn.edu/sentience/SENTIENCE_Vol7. pdf?attachauth=ANoY7coQru26Lg49XTbBaEvIEFJF1915FTfey Yeqt9RVXIwJaFu4rEUBYLM0M4Q-7rDfQCJIxZQclvBVtUe3AIR vDxX_NelahsOAFdnOswSGI6um8wyqj2Kcz3pvRKT2zGLgUKfRSI_84_YwaF75JUMxZ-hwdBwqZs3li-3WvQ2CgDWWUWe EvR7vqp5fnS2EclxfF7QxvSK_vc9L2Jg1T6QIGSfOXpF9g\% 3D\%3D\&attredirects $=0 \#$ page $=6$

Jennings, L. B. (1997). Potential benefits of pet ownership in health promotion. Journal of Holistic Nursing, 15(4), 358-372. https:// doi.org/10.1177/089801019701500404

Killeen, P. R., Fetterman, J. G., \& Bizo, L. A. (1997). Time's causes. In C. M. Bradshaw \& E. Szabadi (Eds.), Advances in psychology. Time and behaviour: Psychological and neurobehavioural analyses (Vol. 120, pp. 79-131). North-Holland/Elsevier Science Publishers. https://doi.org/10.1016/S0166-4115(97)80055-6

Langner, O., Dotsch, R., Bijlstra, G., Wigboldus, D. H., Hawk, S. T., \& Van Knippenberg, A. D. (2010). Presentation and validation of the Radboud Faces Database. Cognition and Emotion, 24(8), 1377-1388. https://doi.org/10.1080/02699930903485076

Le Roux, M. C., \& Kemp, R. (2009). Effect of a companion dog on depression and anxiety levels of elderly residents in a long-term care facility. Psychogeriatrics, 9(1), 23-26. https://doi.org/10. 1111/j.1479-8301.2009.00268.x

Marinho, V., Oliveira, T., Rocha, K., Ribeiro, J., Magalhães, F., Bento, T., Pinto, G. R., Velasques, B., Ribeiro, P., Di Giorgio, L., Orsini, M., Gupta, D. S., Bittencourt, J., Hugo Bastos, V., Teixeira, S. (2018). The dopaminergic system dynamic in the time perception: A review of the evidence. International Journal of Neuroscience, 128(3), 262-282. https://doi.org/10. 1080/00207454.2017.1385614

Martin, R. C., \& Dahlen, E. R. (2005). Cognitive emotion regulation in the prediction of depression, anxiety, stress, and anger.
Personality and Individual Differences, 39(7), 1249-1260. https:// doi.org/10.1016/j.paid.2005.06.004

Mason, j. W. (1975). A historical view of the stress field, part I. Journal of Human Stress, 1(1), 6-12. https://doi.org/10.1080/ 0097840X.1975.9940405

Matell, M. S., Bateson, M., \& Meck, W. H. (2006). Single-trials analyses demonstrate that increases in clock speed contribute to the methamphetamine-induced horizontal shifts in peakinterval timing functions. Psychopharmacology, 188(2), 201-212. https://doi.org/10.1007/s00213-006-0489-x

Pendry, P., \& Vandagriff, J. L. (2019). Animal visitation program (AVP) reduces cortisol levels of university students: A randomized controlled trial. Aera Open, 5(2), 2332858419852592. https://doi.org/10.1177/2332858419852592

Petersson, M., Alster, P., Lundeberg, T., \& Uvnäs-Moberg, K. (1996). Oxytocin increases nociceptive thresholds in a long-term perspective in female and male rats. Neuroscience Letters, 212(2), 87-90. https://doi.org/10.1016/0304-3940(96)12773-7

Petersson, M., Uvnäs-Moberg, K., Nilsson, A., Gustafson, L. L., Hydbring-Sandberg, E., \& Handlin, L. (2017). Oxytocin and cortisol levels in dog owners and their dogs are associated with behavioral patterns: An exploratory study. Frontiers in Psychology, 8, 1796. https://doi.org/10.3389/fpsyg.2017.01796

Polheber, J. P., \& Matchock, R. L. (2014). The presence of a dog attenuates cortisol and heart rate in the Trier Social Stress Test compared to human friends. Journal of Behavioral Medicine, 37(5), 860-867. https://doi.org/10.1007/s10865-013-9546-1

Rattat, A. C., \& Droit-Volet, S. (2012). What is the best and easiest method of preventing counting in different temporal tasks? Behavior Research Methods, 44(1), 67-80. https://doi.org/10. 3758/s13428-011-0135-3

Selye, H. (1956). The stress of life. McGraw-Hill.

Siegel, J. M. (1990). Stressful life events and use of physician services among the elderly: The moderating role of pet ownership. Journal of Personality and Social Psychology, 58(6), 1081. https://doi.org/10.1037//0022-3514.58.6.1081

Tian, Y., Liu, P., \& Huang, X. (2018). The role of emotion regulation in reducing emotional distortions of duration perception. Frontiers in Psychology, 9, 347. https://doi.org/10.3389/fpsyg.2018.00347

Tipples, J. (2008). Negative emotionality influences the effects of emotion on time perception. Emotion, 8(1), 127-131. https://doi. org/10.1037/1528-3542.8.1.127

Tipples, J. (2011). When time stands still: Fear-specific modulation of temporal bias due to threat. Emotion, 11(1), 74-80. https://doi. org/10.1037/a0022015

Treisman, M. (1963). Temporal discrimination and the indifference interval: Implications for a model of the "internal clock". Psychological Monographs: General and Applied, 77(13), 1-31. https://doi.org/10.1037/h0093864

Treisman, M. (2013). The information-processing model of timing (Treisman, 1963): Its sources and further development. Timing \& Time Perception, 1(2), 131-158. https://doi.org/10.1163/2213446800002017

Viau, R., Arsenault-Lapierre, G., Fecteau, S., Champagne, N., Walker, C. D., \& Lupien, S. (2010). Effect of service dogs on salivary cortisol secretion in autistic children. Psychoneuroendocrinology, 35(8), 1187-1193. https://doi.org/10.1016/j. psyneuen.2010.02.004

Wearden, J. (2016). The psychology of time perception. Springer.

Wearden, J. H., \& Ferrara, A. (1995). Stimulus spacing effects in temporal bisection by humans. The Quarterly Journal of Experimental Psychology, 48(4), 289-310. https://doi.org/10.1080/ 14640749508401454

Zasloff, R. L., \& Kidd, A. H. (1994). Loneliness and pet ownership among single women. Psychological Reports, 75(2), 747-752. https://doi.org/10.2466/pr0.1994.75.2.747 


\section{History}

Received November 27, 2020

Revision received June 12, 2021

Accepted June 14, 2021

Published online August 18, 2021

\section{Acknowledgments}

The authors thank Amandine Parakian who was research assistant and collected a large proportion of the participants' data.

\section{Publication Ethics}

Informed written consent was obtained from all participants prior to participation in Experiment 1.

\section{Open Data}

To the best of my ability and knowledge, I have provided all original materials and clear references to all other materials via a stable online repository. The following link gives access to raw data for Studies 1 and 2: https://osf.io/nehs4/?view_only=0ce9a765c1c442 faa863b290f943fc28. We adhered to the preregistered statistical hypotheses and analysis plans.

\section{ORCID}

Quentin Hallez

(iD) https://orcid.org/0000-0003-2035-8694

\section{Quentin Hallez}

Department of Psychology

University Lumiere Lyon 2

5 Avenue Pierre Mendes France

69500 Bron

France

quentin.hallez@gmail.com 